\title{
¿Prevenir, atender y erradicar? Políticas públicas contra la violencia de género en la Ciudad de Buenos Aires, 2015 - 2017.
}

Prevention, assistance, eradication? Public Policies against gender-oriented violence in Buenos Aires City, 2015-2017.

Celina Penchansky

Observatorio de Géneros y Políticas Públicas de la Ciudad

mc.penchansky@gmail.com

Lucía Fuster Pravato

Observatorio de Géneros y Políticas

Públicas de la Ciudad

luciafusterpravato@gmail.com

\section{Victoria Freire}

Observatorio de Géneros y Políticas Públicas de la Ciudad

victoriafreire85@gmail.com

Lucía Loewy

Observatorio de Géneros y Políticas

Públicas de la Ciudad

luloewy@gmail.com

Fecha de recepción:

20.9.17

Fecha de aceptación:

15.12.17

\section{Resumen}

El presente artículo busca dar cuenta del estado de situación de la violencia de género en la Ciudad Autónoma de Buenos Aires y las políticas públicas explícitas llevadas a cabo por el Gobierno de la ciudad para intervenir, prevenir y atender esta problemática. En primer lugar, como objetivo descriptivo pretendemos dar cuenta de la magnitud de este fenómeno. En segundo lugar, nos proponemos realizar un diagnóstico de las principales acciones del gobierno de la CABA contra la violencia de género y de los dispositivos con los que cuenta, en particular la Dirección General de la Mujer y las políticas que de este organismo se desprenden. En tercer lugar, el diagnóstico de las políticas públicas generadas para combatir la violencia hacia las mujeres en la CABA nos permite arribar a algunas inferencias con respecto al tratamiento de la violencia de género desde el Gobierno de la Ciudad Autónoma de Buenos Aires (GCABA). El período elegido para el diagnóstico y el análisis cubre 2015 hasta el último trimestre de 2017. Consideramos pertinente utilizar el año 2015 como referencia ya 
que en ese año se publica el "Registro Nacional de Femicidios de la Justicia Argentina”, donde por primera vez se conocen datos oficiales sobre los femicidios en la República Argentina. A su vez, 2015 es un año fundamental, ya que se realizó la multitudinaria concentración de $\mathrm{Ni}$ una Menos, que permitió visibilizar la magnitud y estructuralidad de la violencia de género en nuestro país. La hipótesis que guía esta investigación es que el GCABA realiza un tratamiento deficiente de la violencia de género, ya que plantea una perspectiva fundamentalmente de asistencia y, a su vez, de manera muy limitada en su alcance. La principal razón de ello es la falta de presupuesto. Esto se traduce en que las políticas públicas no resuelven el problema al que se intenta dar respuesta y dejan por fuera de su intervención distintas aristas que son constitutivas de la violencia de género.

Palabras clave: violencia de género - violencia contra las mujeres - Ciudad Autónoma de Buenos Aires - Dirección General de la Mujer - "Ni una menos"

\section{Abstract}

This article entails an assessment of gender oriented violence in Buenos Aires City and of current public policies addressed at it, focusing mainly on violence against women. In the first place we assess the magnitude of the phenomenon; then we focus on the variety of actions implemented by the City government and their main institutional tools. In a third moment attention given to specific public policies allows us to propose an interpretation of the prevailing policy-approach conducted from the City government. The scope of our discussion goes back to 2015 for two main reasons: 1) the enactment, in that year, of the National Register of Femicides, which merges information from the judicial system as well research conducted by NGOs dealing with violence against women; 2) The impressive massive public mobilization "Not one woman less/Ni una menos" of June 3, that year. Our main conclusion points to the prevalence of assistentialism 
as a policy approach, which in turn we relate to restrictions in resource endowments which prevent the implementations of policies of greater complexity.

Key-words: gender-oriented violence - violence against women - Buenos Aires City - General Directorate for Women - "Not one less"

\section{Introducción}

Según la Unidad Fiscal Especializada en Violencia Contra las Mujeres (UFEM) dependiente del Ministerio Público Fiscal, en la Ciudad Autónoma de Buenos Aires en 2016 se produjeron 18 homicidios dolosos de mujeres, 13 de los cuales fueron femicidios (UFEM, 2017). Lamentablemente, estos datos no nos resultan ajenos, pero es de llamar la atención, ya que la Ciudad de Buenos Aires es una de las localidades del país con una de las legislaciones más progresistas en temas de género y con mayor presupuesto; sin embargo, en ella se verifican profundas desigualdades basadas no sólo en la condición social y económica sino en el género.

Es por ello que en este trabajo nos proponemos indagar la magnitud de la violencia de género en la Ciudad Autónoma de Buenos Aires (CABA) por un lado, y las políticas públicas explícitas que se llevan a cabo por el Gobierno de la ciudad para prevenir, atender y erradicar esta problemática.

En primer lugar, realizaremos precisiones teóricas acerca de la violencia de género y las políticas públicas para luego describir la magnitud de este fenómeno mediante la utilización de datos secundarios obtenidos tanto por organizaciones no gubernamentales (ONGs) como por la Corte Suprema de Justicia de la Nación, teniendo en cuenta que no existen datos oficiales sistemáticos disponibles por parte del gobierno de la CABA, a excepción del año 2016. En segundo lugar, nos proponemos realizar un análisis sobre las acciones estatales en la CABA para prevenir, atender y erradicar la violencia de género, atendiendo particularmente a los dispositivos con los que cuenta la Dirección General de la Mujer, por ser el organismo más relevante para tratar esta problemática, y las políticas que de este organismo se desprenden durante el período mencionado. En tercer lugar, proponemos un diagnóstico de las políticas públicas generadas para combatir la violencia hacia las mujeres en la CABA que nos permite arribar a algunas explicaciones sobre el tratamiento de la violencia de género desde el gobierno local. 
Nuestra hipótesis es que el tratamiento que realiza el Gobierno de la CABA de la violencia de género es deficiente ya que no sólo no se destinan los recursos presupuestarios necesarios para intervenir en esta cuestión, sino que al no ser un abordaje integral no da respuestas a los objetivos que se plantea; en este sentido, al tratarse de políticas que se dedican fundamentalmente a la asistencia, no contribuyen a la prevención ni logran reducir los índices de violencia de género.

Con el objetivo construir un corpus de material a analizar nos propusimos obtener datos sobre los índices de violencia de género en la Ciudad Autónoma de Buenos Aires. Para ello hicimos un relevamiento de datos secundarios públicamente disponibles. Estas fuentes fueron la Encuesta realizada entre el Equipo Latinoamericano de Justicia y Género (ELA) y el Gobierno de la CABA, la encuesta realizada por el colectivo Ni Una Menos y los datos publicados por la UFEM (Unidad Fiscal Especializada en Violencia contra las Mujeres) del Ministerio Público Fiscal. Luego de obtener los datos disponibles sobre los índices, tipos y extensión de la violencia de género en la CABA, nos propusimos indagar la estructura del principal órgano estatal para atender, prevenir y erradicar la violencia de género y las políticas públicas que a través de dicho organismo se llevan a cabo. Para ello realizamos una combinación de producción de datos a través de entrevistas a informantes claves, análisis del presupuesto y relevamiento de la información pública disponible en las propias comunicaciones institucionales de la Dirección General de la Mujer. Este corpus construido nos permitió, luego, realizar un diagnóstico y algunas reflexiones sobre el modo en el cual en GCBA aborda la violencia de género en la ciudad.

\section{Precisiones sobre la violencia de género y las políticas públicas}

La violencia de género es un componente estructural de la opresión de género; no sólo opera como dispositivo de disciplinamiento y control de los cuerpos y las subjetividades, sino que expulsa y/o limita a la mitad de la población de los derechos ciudadanos. Lo que está implícito en este tipo de violencia son las relaciones de poder desigual de un sistema basado en la supremacía del varón, blanco y heterosexual. Tal como señala la antropóloga Rita Segato "los crímenes del patriarcado o feminicidios son, claramente, crímenes de poder, es decir, crímenes cuya función es, en este modelo, simultáneamente, la retención o manutención, y la reproducción del poder” (Segato, 2003: 4).

Estas aseveraciones respecto del acceso diferencial a la ciudadanía, consecuencia del género como vector determinante de la desigualdad no ha formado parte del marco teórico e interpretativo de investigaciones ni de políticas públicas desde siempre. Tanto a nivel nacional como a nivel internacional, gracias al activismo del movimiento feminista y de mujeres, las problemáticas de género han logrado instalarse en la agenda pública y luego en la agenda estatal como problemática. Como lo resalta la investigadora Virginia 
Guzmán los “avances y logros de esta institucionalidad no tienen raíces sólo al interior del Estado sino que también se anclan en los cambios económicos, sociales y culturales en curso en los distintos países de la región” (Guzman, 2001: 33).

La violencia hacia las femineidades - con este término nos referimos a mujeres, travestis y trans- se convirtió en un problema social y político, y tanto el Estado como el movimiento de mujeres y feminista, llevaron a cabo numerosas acciones para el reconocimiento y tratamiento de la problemática. Sin embargo, a pesar de los avances obtenidos, la violencia dirigida a mujeres, travestis y trans es un fenómeno que persiste y se agudiza (Femenías y Rossi, 2009). En este sentido, las respuestas estatales no sólo han sido insuficientes y limitadas, sino que están muy lejos de cumplir con los compromisos asumidos por el Estado Nacional al ratificar la Convención Interamericana para Prevenir, Sancionar y Erradicar la Violencia contra la Mujer,-conocida como la Convención de Belem do Pará-, mediante la ley N²4.632 (Chejter, 2009).

El proceso de construcción de la violencia de género como problema estatal implicó la puesta en evidencia de distintas posiciones (Oszlak y O’Donnell, 1982) por parte de los actores estatales como no estatales; tales concepciones quedaron cristalizadas en diversos abordajes y políticas. Desde la vuelta a la democracia en Argentina, pero con mayor magnitud y celeridad en los últimos años, la violencia ha dejado de considerarse un problema intrafamiliar o doméstico, para ser definido como una violación a los derechos humanos y, por lo tanto, un tema fundamental a ser atendido en las políticas públicas (Sagot, 2008). Estos avances se han constituido desde la agenda pública a la agenda institucional (Luis Aguilar, 1993; Cobb y Elder, 1984; Cobb, Ross y M.H. Ross, 1976; Muller, 1990 citados por Guzmán, 2001), donde las sanciones de nuevas legislaciones son el resultado explícito de una nueva correlación de fuerzas y de nuevos marcos de inteligibilidad. En palabras de las investigadoras Laura Pautassi y Corina Rodríguez:

Vivir una vida libre de violencia es un derecho humano de las mujeres $y$ de las niñas $y$ ha sido convertido en obligación por parte de los países que han ratificado la Convención Interamericana para prevenir, erradicar y sancionar la violencia contra las mujeres (Convención de Belem do Pará), la Convención internacional contra la discriminación de la Mujer (CEDAW) y su Protocolo Facultativo, entre tantos otros instrumentos internacionales. Argentina es uno de los países que voluntariamente ha ratificado estos instrumentos (...), por lo tanto ha asumido las obligaciones de hacer y de no hacer en relación con garantizar este derecho

(Pautassi y Rodriguez, 2016: 41). 
De este modo, la ley 26.485, para prevenir, sancionar y erradicar la violencia de género, sancionada en 2009, implica un importante avance producido por los movimientos de mujeres y feministas para visibilizar y atender esta problemática en nuestro país. La ley define a la violencia de género como "toda conducta, acción u omisión, que de manera directa o indirecta, tanto en el ámbito público como en el privado, basada en una relación desigual de poder, afecte su vida, libertad, dignidad, integridad física, psicológica, sexual, económica o patrimonial, como así también su seguridad personal". Es decir, reconoce que se trata de una violencia basada en relaciones desiguales de poder derivadas del género y que afecta la vida de las mujeres, travestis y trans en los diferentes ámbitos donde ellas transcurren: dimensiona la violencia es su carácter integral, transversal, físico, emocional y patrimonial y, hace responsable al Estado de su atención, prevención y erradicación.

Por consiguiente, bajo esta perspectiva, toda política estatal es esencialmente, una toma de posición del Estado frente a una determinada cuestión socialmente problematizada; toma de posición que tanto puede ser acciones como omisiones (Oszlak y O’Donnell, 1982). Ello implica que es sólo una parte de un proceso social más abarcativo, que incluye una multiplicidad de actores sociales además de los aparatos del Estado implicados, los cuales toman posición en torno al problema-motivo de la política pública.

Por lo tanto el estudio de las políticas estatales es el estudio de un proceso, de una secuencia de tomas de posición, de un conjunto de acciones y omisiones por parte del Estado y los actores que movilizan la cuestión o están interesados en ella. En este caso específico, el movimiento de mujeres y las organizaciones feministas fueron piezas clave para la visibilización y la cristalización de la violencia de género en la agenda del Estado argentino, siendo la ley 26.485 ejemplo de ello.

El Estado proporciona a la sociedad su tejido conectivo a través de las políticas públicas y las tomas de posición (Oszlak 2012). Agregamos nosotras que no es el único componente de dicho tejido conectivo pero que sí determinante. Es decir que el Estado está presente (o ausente) tanto a través de aquello que prohíbe o sanciona como de los riesgos que previene y las oportunidades que crea (o niega) en la vida cotidiana de las personas. En este sentido, Oszlak resalta que el Estado puede ser observado a partir de tres niveles: el macro, el meso y el micro. El nivel micro de las políticas públicas sería aquello que las personas experimentan en su vida cotidiana: tener un Centro Integral de la Mujer en el barrio, tener acceso al patrocinio jurídico, acceder a subsidios habitacionales y políticas de promoción del empleo o no acceder. Sin embargo, la afectación de la vida cotidiana de las mujeres y las disidencias sexo-genéricas también se ven modificadas por aquello que el Estado hace en los niveles macro y meso. Si bien, por cuestiones de espacio no lo desarrollaremos aquí, debe señalarse que los cambios acontecidos en Argentina con la victoria de la Alianza Cambiemos en las elecciones presidenciales de 2015, han producido un deterioro de la capacidad de consumo del salario y las políticas 
represivas han afectado directa y negativamente la posición de las mujeres, travestis y trans en la capacidad de ampliación de sus derechos (Hiller y Jones 2016).

En este sentido el autor plantea que la modalidad, calidad y extensión de las políticas públicas determinan lo que él conceptualiza como la capilaridad social del rol del Estado (Oszlak, 2012). Es decir, la manifestación de su presencia celular en la organización de la vida de una sociedad. De este modo, para abordar el itinerario de violencia, el Estado debe estar presente en múltiples acciones que faciliten a las víctimas de violencia de género la posibilidad de salir del círculo de violencia desde una perspectiva integral. Es decir, que interpele la vida de quien es víctima de violencia de género en las distintas dimensiones: su acceso a la salud, a la vivienda, al patrocinio jurídico y a un trabajo digno.

Las políticas estatales no son genéricamente neutras, sino que inevitablemente inciden en la reproducción o transformación de las relaciones sociales entre los géneros (Rofman, 2000). En palabras de Ana Laura Rodríguez Gustá:

(...) el Estado -aún el democrático- representaría, predominantemente, los intereses de varones, por lo cual sería una institución patriarcal $y$, por tanto, un vehículo signifiativo de la opresión sexual y de la propia regulación de la desigualdad de género (Connell, 1990). A pesar de estas limitaciones, es posible impulsar políticas que fomenten una mayor igualdad de género por la mentada "autonomía relativa” del Estado y, además, porque los intereses usualmente conforman un terreno contradictorio ( $y$ no homogéneo) de preferencias (Haney, 1996). De esta forma, y en combinación con demandas provenientes de grupos de mujeres organizadas insertas en los espacios públicos deliberativos, el Estado podría mejorar las condiciones de vida relativas de ambos sexos.

(Rodríguez Gustá, 2008: 113-114)

En este sentido, mediante el Estado se pueden trastrocar los tejidos conectivos de la sociedad (Oszlak, 2012; Rodríguez Gustá 2008) para brindar soluciones y transformar, mediante las políticas públicas, las relaciones sociales desiguales entre los géneros. Por lo tanto, el costo fiscal no debe ser en ningún sentido el principal argumento para no llevarla a cabo. En palabras de Pautassi y Rodríguez:

La restricción fiscal no puede, en ningún caso, ser una excusa para la inacción del Estado en materia de violencia contra las mujeres. Más aún cuando se demuestra que ac- 
tuar en este sentido resulta económicamente más eficiente que no hacerlo. En todo caso, lo central es reconocer que la violencia contra las mujeres es una violación de derechos humanos y es el Estado el responsable por no impedirla (Pautassi y Rodríguez, 2016: 42).

La responsabilidad estatal, su complicidad por omisión o ineficiencia, es necesario visibilizarla para comprender que para prevenir, sancionar y -más aún- erradicar la violencia de género en nuestra sociedad, es necesario trastrocar las estructuras sociales, los modos de subjetivación y los capitales sociales de los que disponen las víctimas, así como trabajar sobre los modos de subjetivación propios de quienes ejercen la violencia.

Teniendo en cuenta el marco teórico descripto anteriormente, en los próximos apartados nos dedicamos a identificar la magnitud de la violencia de género en la Ciudad de Buenos Aires y a realizar un diagnóstico sobre las políticas públicas que se han destinado para prevenir, atender y en concreto a asistir a las víctimas de violencia de género.

\section{Los números de la violencia en la CABA}

Para realizar una fotografía de la violencia de género en la Ciudad Autónoma de Buenos Aires, nos valimos de datos secundarios. Al respecto es necesario aclarar que hasta 2015 no existían registros oficiales sobre mujeres víctimas de femicidio. Esta deuda por parte del Estado de cuantificar el fenómeno de la violencia de género fue uno de los puntos de reclamo de distintas organizaciones de mujeres por muchos años, refrendándose en la movilización Ni Una Menos de 2015. Cabe destacar que la Asociación civil Casa del Encuentro impulsó los primeros registros de femicidios desde 2008, en base a noticias de 120 diarios de tirada nacional y provincial. Por otro lado, en 2014 el Ministerio de Seguridad de la Nación presentó el Análisis de la violencia de género a través de la gestión de denuncias e investigaciones policiales.

Como resultado de los reclamos de Ni Una Menos, meses más tarde de la primera movilización en junio 2015 se creó el Registro Nacional de Femicidios de la Justicia Argentina, que releva las causas por homicidio de mujeres (niñas, adolescentes y/o adultas) que hayan sido perpetrados por varones por razones asociadas con su género, tipificadas o no como femicidio. En otro orden, que va más allá de los femicidios a la hora de cuantificar la violencia de género, la Oficina deViolencia Doméstica de la Corte Suprema de Justicia de la Nación publica semestralmente desde 2009 los casos que se denuncian, incluyendo la información vinculada a características sociodemográficas requeridas por la ley 26.485. Sobre estos registros y construcción de datos cabe aclarar que no sólo es importante la tarea de confeccionarlos, sino también tener en cuenta la perspectiva de género a la hora de realizar las investigaciones para evitar posibles sesgos sexistas tanto en las encuestas y en 
otros instrumentos de investigación estadística, y para generar que los instrumentos de recolección también puedan relevar la violencia que sufren travestis y trans.

Asimismo, los registros sobre femicidios dan cuenta de la expresión más brutal de la violencia machista, pero no representan la magnitud total de este fenómeno, que como lo define la ley 26.485, abarca otros tipos de violencias. Por otro lado, existen ciertos inconvenientes a la hora de realizar los registros de violencia de género en base a denuncias, ya que sólo tienen en cuenta los casos en los que las mujeres llegan a denunciar o solicitan ayuda a personas de su entorno que denuncian por ellas, lo que invisibiliza al resto de las mujeres que no llegan a hacerlo.

Teniendo en cuenta estos resguardos respecto de aquello que los datos nos permiten identificar, y a pesar de las falencias metodológicas, consideramos pertinente -por su utilidad heurística- reponer los datos construidos por las investigaciones más recientes en relación a la temática.

Según la investigación Argentina cuenta la Violencia Machista (Beck y Romero, 2016), impulsada por activistas feministas y ONGs, la violencia de género en Argentina, en sus distintas expresiones, afecta a 9 de cada 10 mujeres. Esta situación se replica en la Ciudad Autónoma de Buenos Aires, donde el 93\% de la población femenina ha sido víctima de acoso en espacios públicos y privados, el $92 \%$ de aislamiento de la pareja y el $86 \%$ de estigmatización. Es decir, que en la CABA 9 de cada 10 mujeres han sido controladas, celadas y/o cuestionadas en sus vínculos o actividades por parte de su pareja.Y 8 de cada 10 a tenido sentimientos de riesgo o menosprecio por su género. ${ }^{1}$ 


\section{Tipo y extensión de violencia de género, Ciudad Autónoma de Buenos Aires, 2016.}

\section{Ciudad Autónoma de Buenos Aires}

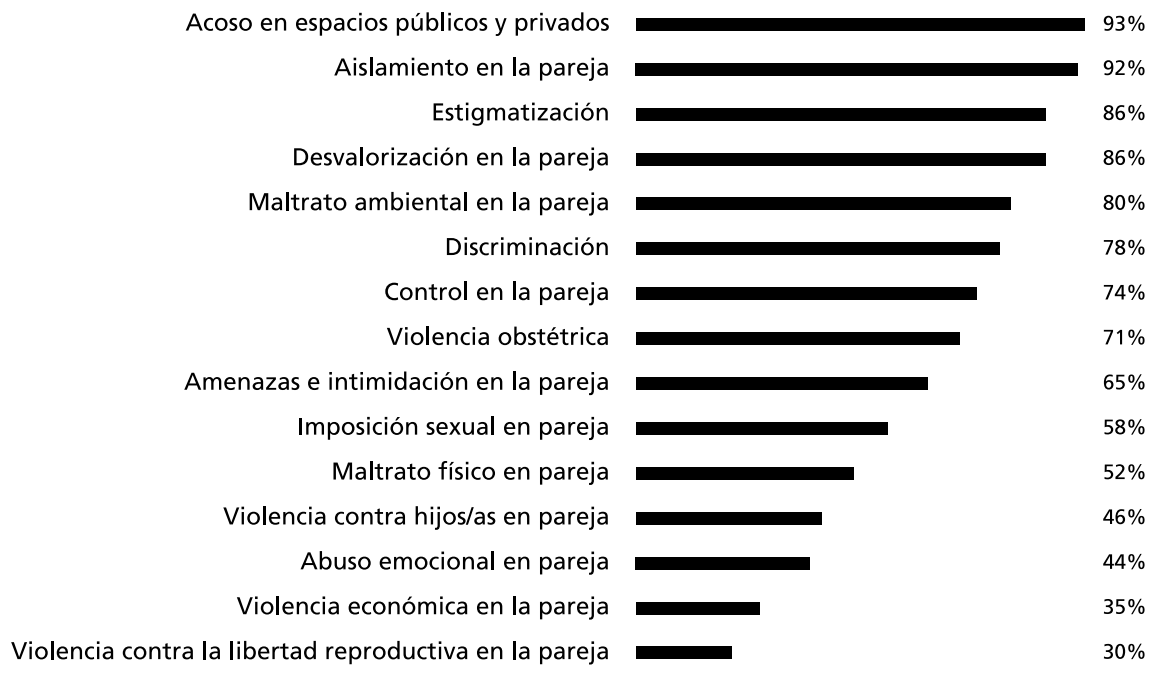

Fuente: Construcción propia a partir del Informe "Argentina cuenta la Violencia Machista"

Base: 19.068 casos de mujeres, trans y travestis residentes en la CABA.

A su vez, estos datos están en consonancia con los resultados arrojados por la investigación llevada a cabo por el Equipo Latinoamericano de Justicia y Género (ELA) en conjunto con el Gobierno de la Ciudad de Buenos Aires en 2016 (ELA-GBA, 2016). La muestra, compuesta por 1003 casos de mujeres residentes en la CABA, atendiendo a los requisitos de representatividad, permite observar que en la ciudad 6 de cada 10 mujeres fue víctima de violencia psicológica y 3 de cada 10 de violencia física/sexual.

Con respecto a estos números, nos parece necesario señalar que las violencias de género que tienen como primeras expresiones la violencia psicológica y física, tienen su máxima expresión en los femicidios. En este sentido, los datos arrojados por la UFEM indican que 94\% de los casos de femicidios perpetrados en la CABA durante el 2014, fueron cometidos dentro del ámbito familiar. En este sentido, también es importante destacar la existencia de una diferencia significativa en torno a la georreferencialidad de los homicidios de hombres y de mujeres en la CABA (no hay datos estadísticos respecto de los asesinatos de travestis y trans). El informe de la UFEM destaca que, según el Ministerio de Seguridad de la CABA, los asesinatos de varones se concentran en barrios donde hay "asentamientos de emergencia" -vinculados a otros núcleos de violencias y 
discriminaciones- mientras que los asesinatos de mujeres se han dado, durante el 2014, en barrios muy disímiles entre sí. Para ese mismo año, la vivienda ha sido el escenario de muerte en el 68\% de los asesinatos de mujeres.

Los sondeos de las investigaciones citadas permiten observar que tanto el ámbito considerado "público" como el considerado "privado" son sitios donde el género aparece como un factor de riesgo para la seguridad individual. Este fenómeno pone de manifiesto lo que el feminismo ha denunciado: la ficcionalidad de la separación público/ privado (Mouffe, 1992) donde la escisión de estos espacios esconde la instalación de su jerarquización y la determinación de los ámbitos de intervención del Estado.

En línea con lo anterior, la expresión "lo personal es político" acuñada por el feminismo permitió poner en cuestión las violencias en el ámbito "doméstico" como aquellas circunscritas al fuero íntimo y personal. El propio término "femicidio" conceptualiza el asesinato que hasta hace unos pocos años se definía como "pasional”, y condensa el entrecruzamiento que implica la violencia de género: el ordenamiento desigual de los roles de géneros en los vínculos interpersonales y familiares.

Asimismo, la separación entre lo público y lo privado ha delimitado los ámbitos que el Estado tradicionalmente ha reconocido como ámbito de intervención. Si bien - tal como observamos con Oszlak- el Estado incide en los tejidos conectivos tanto a partir de aquello que permite como de aquello que niega, la delimitación clásica, sustentada sobre una estructura societal patriarcal del Estado liberal-democrático, ha delimitado hasta hace no muchos años que no interviene en el ámbito instituido como "doméstico" y "privado".

Por esta razón, resaltamos que los resultados que arrojan las últimas investigaciones sobre la violencia machista en la CABA, deben ser tenidos en cuenta por el ejecutivo local y por los dispositivos que construye para responder a esta problemática. Como observamos, los datos que aportan estas investigaciones para cuantificar la violencia machista arrojan que la mayoría de las mujeres sufren distintos tipos de violencias y que por este motivo, las respuestas del gobierno en cuanto a las políticas públicas deben ser integrales. Veremos a continuación que las políticas públicas destinadas a mitigar la violencia de género están destinadas principalmente, aunque de manera deficiente, a la asistencia de las víctimas y lejos se encuentran de brindar un abordaje desde la integralidad, que brinde respuestas a esta compleja cuestión que sufren principalmente las mujeres, mujeres trans y travestis. ${ }^{2}$

Por este motivo, consideramos que para poder abordar la violencia de género cuando ésta está consumada, en primer lugar las opciones de salida deben ser integrales, ingresando en los ámbitos tradicionalmente vinculados a la "domesticidad" y proponiendo alternativas que aborden diferentes aristas afectadas por esta cuestión. En segundo lugar, consideramos que la violencia machista abarca otros tipos de violencia, que van más allá de la violencia fisica y psicológica, y que no son atendidas 
por el GCBA, ya que las acciones están destinadas principalmente a la asistencia de las víctimas. En el próximo apartado realizamos un diagnóstico y análisis de las políticas que se han llevado a cabo para atender a la violencia de género, observando con qué perspectiva han sido diseñadas y cuál es el alcance y los principales puntos débiles que presentan. Nos enfocamos en la Dirección General de la Mujer por ser el órgano estatal principal del cual se desprenden las políticas de asistencia a las víctimas de violencia machista.

\section{La Dirección General de la Mujer}

Hemos señalado ya que la CABA es una de las jurisdicciones del país con la legislación más avanzada en cuestiones de género; no obstante, por los números que hemos podido analizar, en ella se verifican profundas desigualdades de género y altos índices de violencia contra las mujeres.

En este orden de cosas cabe señalar la Ley 474, que crea el Plan de Igualdad Real de Oportunidades y de Trato entre Mujeres y Varones, una acción que se enmarca en lo que la bibliografia sobre políticas públicas con sensibilidad de género reconoce como una política con perspectiva de género. Según Rodríguez Gustá, existen cuatro categorías de políticas sensibles al género:"a) las políticas de acción afimativa,b) las políticas para las mujeres, c) las políticas con perspectiva de género y, finalmente, d) las políticas de transversalización de género -conocidas por su término en inglés gender mainstreaming-"(Rodríguez Gustá, 2008: 110) Las políticas con perspectiva de género buscan mediante su intervención cambiar las relaciones jerárquicas de género.

Además, la CABA adhiere a la Ley Nacional 26.485 y cuenta con otras leyes que señalan acciones positivas para prevenir y atender la violencia de género: la ley 4181 que crea la LÍNEA MUJER, servicio de atención telefónica gratuita permanente dependiente de la Dirección General de la Mujer; la ley 5742 de prevención y sanción del acoso sexual verbal o fisico en espacios públicos o de acceso público, que hostiguen, maltraten o intimiden y que afecten en general la dignidad, la libertad, el libre tránsito y el derecho a la integridad física o moral de personas, basados en su condición de género, identidad y/u orientación sexual.

En este sentido, la Dirección General de la Mujer (DGM), actualmente dependiente de la Subsecretaría de Promoción Social dentro del Ministerio de Hábitat y Desarrollo Humano es el principal dispositivo con el que cuenta el Gobierno de la Ciudad Autónoma de Buenos Aires para llevar a cabo políticas públicas explícitas (Rofman, 2000) contra las desigualdades de género. ${ }^{3}$

La DGM está conformada por dos gerencias operativas compuestas cada una por un programa y por una línea de acción/intervención. La Gerencia Operativa de Atención Integral a Víctimas de Violencia Doméstica y Sexual actúa ante las situaciones de violencia de género ya consumadas. En esta gerencia se inscribe el Programa 69, de "Asistencia a las Víctimas de Violencia". La Gerencia Operativa de Fortalecimiento de 
las Políticas de Igualdad de Oportunidades se centra en la promoción y fortalecimiento de derechos; en ella está inscripto el Programa 70 "Fortalecimiento de las Políticas de Igualdad de Oportunidades"

En el año 2017 el Programa Asistencia a la Víctima representó 79.3\% del presupuesto, y el Programa de Fortalecimiento de Políticas para la Igualdad de Oportunidades (FPIO) 20.7\%. Una situación similar se da con la distribución de recursos humanos, el Programa de Fortalecimiento cuenta con una planta mínima de trabajadoras que además trabajan en más de un sub-programa, con lo cual sus acciones tienen un impacto limitado.

Con respecto a la asignación presupuestaria y tal como planeta Morales (2015), los derechos suponen la necesidad de asignación de recursos para poder ser traducidos en políticas públicas concretas. ${ }^{4}$ En este sentido esta breve descripción de la organización de la DGM pone al descubierto la concepción subyacente de las políticas de género que se aplican en la ciudad. La distribución del presupuesto y del personal muestra que la DGM prioriza la atención a las situaciones de violencia ya consumadas y en menor medida se trabaja sobre el fortalecimiento y la promoción de los derechos. A pesar de ello, los dispositivos existentes para intervenir allí donde la violencia de género ya ha sido consumada, son limitados: tanto en términos de capilaridad y accesibilidad, así como también desde la integralidad.

\section{Programa Asistencia a las Víctimas de Violencia}

El Programa “Asistencia a las Víctimas deViolencia” cuenta con tres tipos de dispositivos de atención físicos y un dispositivo de atención telefónica. Los dispositivos de atención físicos son: los Centros Integrales de la Mujer (CIMs), las Casas de Medio Camino y los Refugios de Alta Seguridad. 


\section{Estructura organizacional del Programa Asistencia a Víctima de Violencia, Dirección General de la Mujer, GCBA, 2017.}

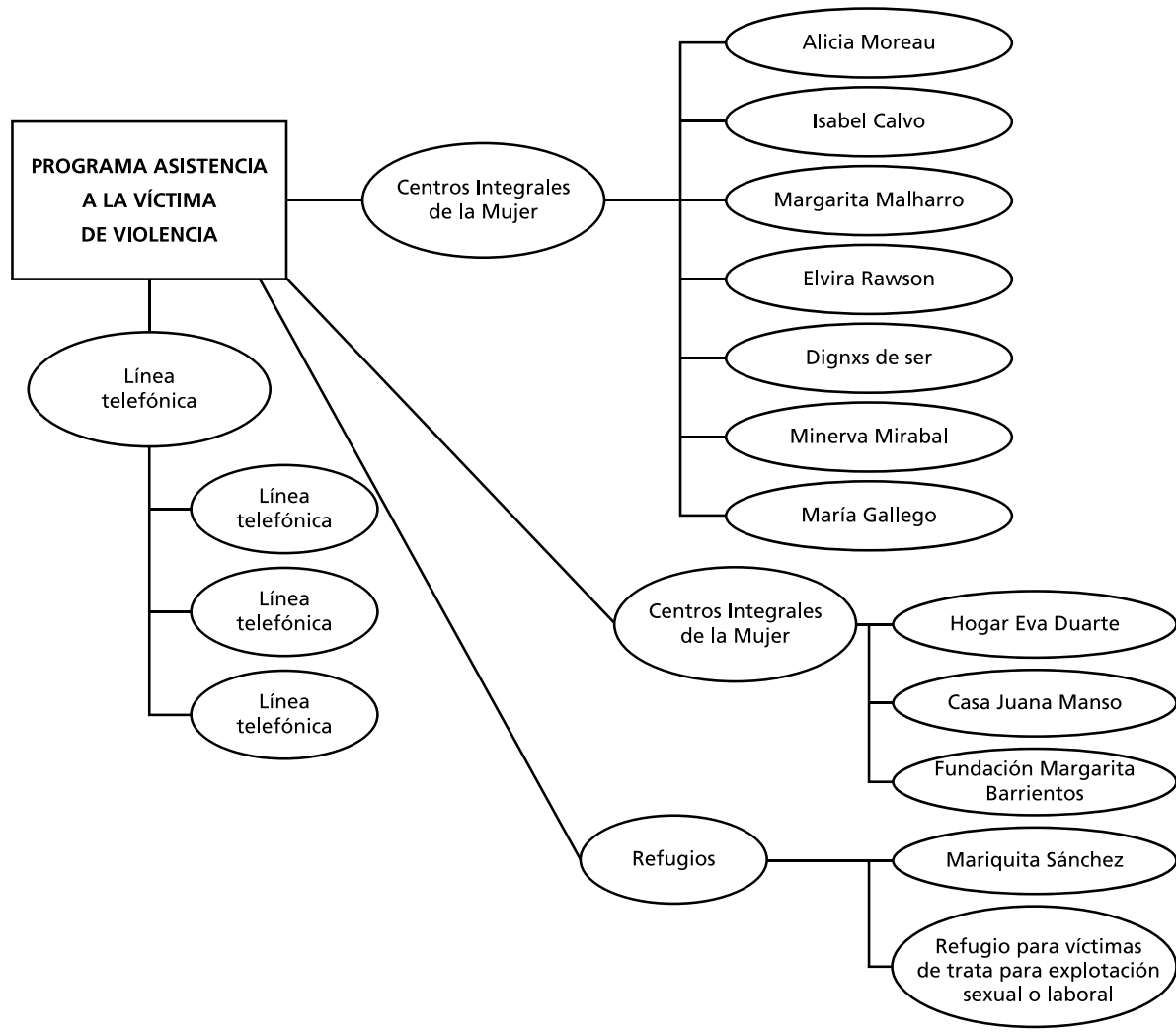

Fuente: Producción propia en base a los datos obtenidos en: http://www.buenosaires.gob.ar/desarrollohumanoyhabitat/institucional-subsecretaria-de-promocion-social/dg-mujer

Los CIMs son un tipo de dispositivo de atención territorial que opera a partir de la demanda espontánea y están localizados en distintos barrios. Brindan contención, acompañamiento y asesoría psicológica y jurídica a las mujeres mayores de 18 años víctimas de violencia de género.

La ciudad cuenta con ocho CIMs, de los cuales siete corresponden al Programa "Asistencia a Víctimas de Violencia" y el octavo depende del Programa "Fortalecimiento de Políticas de Igualdad de Oportunidades”. Según las disposiciones gubernamentales cada uno de los CIMs debe estar conformado por un equipo interdisciplinario, compuesto por un/a abogado/a, un/a trabajador/a social y un/a psicóloga, lo que no siempre se cumple como veremos en el cuadro a continuación, teniendo en cuenta que la mayor parte del presupuesto de la DGM se destina a la asistencia de las víctimas. 
Cuadro resumen. Centros Integrales de la Mujer: Localización, Horarios de Atención y Composición del Equipo Interdisciplinario

\begin{tabular}{|c|c|c|c|}
\hline & \multicolumn{3}{|c|}{ Programa "Asistencia a Víctimas de Violencia" } \\
\hline & Ubicación & Horario de atención & Equipo interdisciplinario \\
\hline CIM Alicia Moreau & Comuna 1 & Lun aVie de 12.00 a $19.00 \mathrm{hs}$ & SI \\
\hline CIM Isabel Calvo & Comuna 1 & Lun a Vie de 10.00 a $16.00 \mathrm{hs}$ & NO (sólo abogada y psicóloga) \\
\hline CIM Margarita Malharro & Comuna 3 & Lun a Vie $24 \mathrm{hs}$ & NO (sólo abogada y psicóloga) \\
\hline CIM Elvira Rawson & Comuna 5 & Lun aVie de 12.00 a $19.00 \mathrm{hs}$ & NO (sólo abogada y psicóloga) \\
\hline CIM Dignxs de ser & Comuna 7 & Lun a Vie de 13.00 a 20.00 hs & SI \\
\hline CIM Minerva Mirabal & Comuna 8 & Lun a Vie de 8.30 a $15.30 \mathrm{hs}$ & SI \\
\hline CIM Maria Gallego & Comuna 11 & Lun a Vie de 8.00 a 17.00 hs & NO (solo abogada y psicóloga) \\
\hline
\end{tabular}

A partir de los dispositivos existentes, se observa el incumplimiento de la ley 5466, que desde diciembre de 2015 dispuso la creación de un CIM por comuna y se propuso llegar a contar con uno de estos dispositivos cada 50 mil habitantes mujeres por comuna. En la actualidad, sobre un total de 15 comunas, sólo 6 cuentan con Centros Integrales para la Mujer y, entre ellos, sólo 3 con el equipo interdisciplinario completo. Además, ninguno atiende los fines de semana.

A su vez, teniendo en cuenta que el objetivo de la ley 5466 es alcanzar un CIM cada 50 mil mujeres, estos números están cerca de cumplirse sólo en la comuna 1, la única que cuenta con dos CIM. La Comuna 3 también cumple ese número si contemplamos el CIM que depende del Programa "Fortalecimiento de Políticas de Igualdad de Oportunidades"; sin embargo, es un CIM que tiene otras funciones y objetivos. Las comunas 5 , 7, 8 y 11 sólo tienen un CIM cada 100 mil mujeres aproximadamente. ${ }^{5} \mathrm{Y}$ las comunas $2,4,6,9,10,12,13,14$ y 15 no cuentan con ninguno de dichos centros. Estos datos son de suma relevancia teniendo en cuenta la complejidad de algunas de las comunas para poder acceder a bienes y servicios públicos. Si bien, como vimos anteriormente, la violencia de género no tiene una georreferencialidad específica, sino que es transversal, sí es relevante destacar que los niveles de pobreza, inciden en las posibilidades de tender redes para salir del círculo de la violencia. La falta de empleo y los limitados recursos económicos son dimensiones de mucho peso para poder acceder a los dispositivos existentes, sobre todo cuando éstos quedan lejos del hogar de las víctimas.

De acuerdo al Anuario de Derechos Económicos, Sociales y Culturales de la CABA, publicado por el Ministerio Público de Defensa (MPD, 2015: 14-15), existe una distribución desigual de los déficits en el territorio, lo que genera una ciudad desigual, con acceso diferencial a las políticas públicas. La reducida amplitud de horarios de atención y la falta de CIMs por comuna exponen la limitada capilaridad del Estado en términos de políticas públicas que busquen promover la igualdad de género y erradicar la violencia machista. 
Dentro de los CIMs también funcionan los subprogramas "Hombres Violentos", "Asistencia al Maltrato Infantil - CIENA", "Delitos contra la integridad sexual", "Lazos de Mujer", "Noviazgos violentos" y "Grupo de ayuda mutua". Estos subprogramas carecen de personal suficiente. Por ejemplo, el subprograma "Noviazgos Violentos" cuenta con sólo 2 psicólogas para toda la CABA. La escasa cantidad de trabajadoras asignadas evidencia el limitado impacto de una política que trabaja con los vínculos violentos desde la juventud. Además los subprogramas "Hombres Violentos”, "Asistencia al Maltrato Infantil" y "Noviazgos Violentos", funcionan -al menos en uno de los días de atención- dentro de la estructura del CIM Isabel Calvo (Piedras 1281), que carece de la infraestructura necesaria para poder operar correctamente, sobre todo por la falta de espacios con la correcta privacidad para abordar los temas que allí se desarrollan.

A los problemas identificados en los Centros Integrales de la Mujer se agrega una disminución de la cantidad de mujeres asistidas en ellos, a pesar del aumento de la visibilización y recrudecimiento de la violencia machista. Desde el año 2002 hasta el 2015, 136.468 mujeres víctimas de violencia han sido han sido atendidas en los CIM, 2.605 mujeres en promedio por año. Sin embargo, es un dato a considerar que desde el 2011 -año en que esta atención alcanzó su máximo de mujeres atendidas- la cifra ha ido disminuyendo hasta alcanzar su mínimo en 2015, con una atención de 1.955 mujeres víctimas de violencia.

\section{Mujeres víctimas de violencia de género asistidas en los Centros Integrales de la Mujer. 2002-2015}

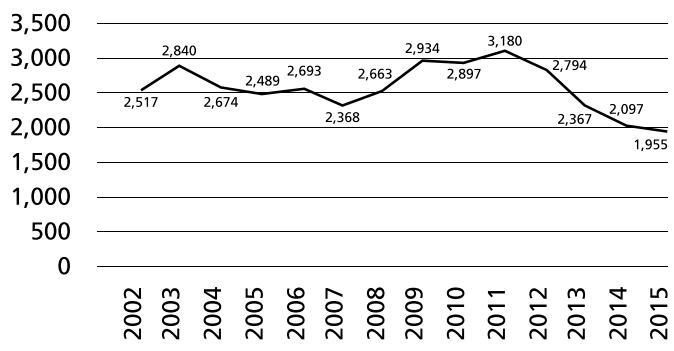

Fuente: Elaboración propia en base a los datos del Banco de Estadísticas y Censos del GCBA.

Llama la atención que en un contexto en el que asistimos a una mayor visibilización de la violencia de género en sus distintas manifestaciones, en la DGM las cifras muestran una merma en la atención de mujeres. La deficiencia en el funcionamiento de los CIM y su capacidad para llegar a la población destinataria puede ser un factor explicativo de dicha merma; otro factor puede ser la escasa difusión de las políticas tanto de asistencia a las víctimas como de igualdad de oportunidades, ya que según hemos visto en el apartado sobre los números de la violencia, ésta se encuentra muy extendida en la sociedad. Por otro lado, el hecho de que la DGM se vea afectada por la disminución de 
su presupuesto y que los CIMs carezcan de personal tanto administrativo como profesional para atender a las mujeres que se presenten, y que la infraestructura de estos no sea la adecuada para la magnitud de la problemática, da cuenta de la jerarquización que el GCBA otorga a la prevención y atención de la violencia machista.

Además de los CIM, el Programa de "Asistencia a la Víctima de Violencia" cuenta también con otros dos tipos de dispositivos físicos, las Casas de Medio Camino y los refugios o albergues. Las Casas de Medio Camino son dispositivos de alojamiento de puertas abiertas, lo que significa que las mujeres, niños y niñas que allí se alojan pueden entrar y salir para sus quehaceres cotidianos. Las mujeres pueden acceder en caso de encontrarse en situaciones de vulnerabilidad y deben ser derivadas por la Oficina de Violencia Doméstica de la Corte Suprema de Justicia de la Nación (OVD). Actualmente existen 3 casas con estas características: el Hogar "Eva Duarte", la Casa "Juana Manso" y la Casa de Medio Camino "Fundación Margarita Barrientos".

El Hogar Eva Duarte se dedica a la asistencia y contención de niñas y adolescentes madres o embarazadas menores de 18 años. Es un dispositivo muy particular dentro de la estructura de la DGM, ya que trabaja con una población que articula más con las dependencias de Niñez, Adolescencia y Juventud que con las propias estructuras de la DGM. Según la información que hemos podido recabar, este dispositivo se enfrenta a una demanda que no puede contener y una reducción de personal que dificulta la posibilidad de trabajo de calidad con las jóvenes allí alojadas y con sus niños/as. Además, si bien la situación edilicia del hogar no es mala, el hecho de que esté ubicado en un segundo piso y sin ascensor, complica la movilidad de las mujeres embarazadas y/o con niños/as pequeños/as.

La Casa Juana Manso y la Casa "Fundación Margarita Barrientos” son las únicas dos instituciones de medio camino que tienen como política explícita funcionar como albergue para las mujeres y sus niños y niñas en situación de riesgo de nivel medio. Ambas tienen como población objetivo a mujeres en situación de violencia de género, sus hijas sin límite de edad y sus hijos hasta los 11 años. El tiempo protocolar de permanencia en este dispositivo es de tres meses, ya que se parte de la noción de que una vez afuera del círculo de violencia la mujer y su familia pueda tender nuevas redes. Sin embargo, las mujeres en condiciones de violencia de mediano riesgo llegan con una situación compleja en términos vinculares, laborales y de seguridad personal. Con lo cual tienden a quedarse más tiempo, teniendo un promedio de permanencia entre uno y dos años. Luego de haber salido de la situación de riesgo de violencia inminente, la permanencia en el hogar responde a la falta de integralidad de políticas públicas que le permitan a las mujeres tener herramientas para desarrollar una vida autónoma: políticas habitacionales, políticas de empleo, disponibilidad de los servicios de cuidado de menores y adultos/as a cargo, por ejemplo. 
La Casa Juana Manso cuenta con una capacidad para albergar aproximadamente 50 personas y la planta laboral está compuesta por 21 trabajadoras, entre el equipo profesional, las facilitadoras/operadoras y las coordinadoras de la casa. Al igual que los demás dispositivos, presenta falta de personal, que se traduce en que muchas veces las guardias dependan de una sola trabajadora con más de 40 personas a cargo. Por su parte, la Fundación Margarita Barrientos es la primera casa abierta bajo la actual gestión gubernamental de Cambiemos; al igual que el primer CIM abierto, tuvo lugar en articulación con una organización no gubernamental. Esta casa tiene una capacidad de albergue de aproximadamente 30 personas; carece de atención psicológica: las personas necesitadas de ella son derivadas para su atención a los Centros Integrales de la Mujer.

A diferencia de las casas de Medio Camino, los refugios de alta seguridad, son dispositivos de puertas cerradas, donde quienes acceden a los mismos no pueden salir y entrar para realizar sus quehaceres cotidianos. Esto supone, a su vez, la confidencialidad de sus emplazamientos y el aislamiento total. Actualmente existen 2 dispositivos de estas características que atienden a diversa población objetivo: el refugio "Mariquita Sánchez" y el refugio para víctimas de trata. El primero es un dispositivo de albergue para mujeres y sus familias con un grado de riesgo alto. Cuenta con una capacidad para albergar a 35/40 personas en términos de espacios y de camas disponibles. Sin embargo se ve permanentemente desbordado por la falta de condiciones edilicias (poca cantidad de baños, falta de mesas, sillas, espacios de recreación). Es importante resaltar que a este refugio llegan a través de la derivación de la OVD bajo la carátula de "Alto riesgo", lo cual implica que la situación de la mujer y la familia es sumamente delicada y necesita de un espacio adecuado para poder salir de la situación de violencia y del shock post traumático. A su vez sucede que, consecuencia del alto riesgo de vida, las solicitudes de ingreso al refugio no son rechazadas, puesto que es el único dispositivo de alta seguridad.

El Refugio Para Víctimas de Trata Para Explotación Sexual o Laboral, tal como lo indica su nombre tiene como objetivo trabajar con la población recuperada de las redes de trata. Sin embargo, de este refugio no hemos podido obtener información que dé cuenta del estado de situación edilicia, de la planta trabajadora ni de su trabajo con la población objetivo.

Teniendo en cuenta este diagnóstico, en el siguiente apartado brindamos algunas hipótesis explicativas de la falta de efectividad de las políticas públicas ideadas desde el GCBA para responder a la problemática de la violencia de género. Consideramos que gran parte de los problemas que presentan estas políticas está íntimamente relacionada a la falta de presupuesto y jerarquización por parte del gobierno local, a la impronta de asistencia que las caracterizan y a la falta de una perspectiva de integralidad que atienda a todas las posibles aristas de la violencia machista. 


\section{Consideraciones sobre el estado de situación de la Dirección General de la Mujer}

La indagación sobre el estado de situación de la violencia de género en la CABA y las políticas públicas explícitas llevadas a cabo por el Gobierno de la Ciudad, nos permiten arribar a algunos diagnósticos preliminares respecto de la Dirección General de la Mujer y los programas y políticas que se desprenden de este organismo durante el período temporal señalado.

En primer lugar, identificamos que las políticas públicas llevadas a cabo a través de la DGM parten de un perfil principalmente de asistencia ante las violencias de género ya consumadas. A partir del organigrama interno de la institución, se observa que tanto en términos del personal como de recursos económicos, el programa de Asistencia a laVíctima es el prioritario. Es decir, que el principal dispositivo para llevar a cabo políticas explícitas que generen nuevo tejidos conectivos en la sociedad en términos de igualdad de géneros, trabaja sobre la violencia desde la asistencia. Sin dudas es de suma urgencia la necesidad de construir respuestas rápidas, integrales y con perspectiva de género para las víctimas. Sin embargo, es necesario trabajar sobre la promoción de la igualdad y el fortalecimiento de derechos en las situaciones donde la violencia no ha sido consumada. En este sentido, consideramos fundamental elaborar políticas públicas desde la perspectiva de género, es decir las que "buscan distribuir recursos materiales y simbólicos con el fin de ampliar las oportunidades de elección de las mujeres, por lo cual la transformación de las relaciones jerárquicas de género constituye el núcleo fundamental de intervención” (Rodríguez Gustá, 2008: 113).

A pesar de ser el programa "Asistencia a la Víctima" el principal dispositivo de intervención, se identifica la falta de despliegue territorial. Esto se observa en que nueve de las quince comunas carecen de Centros Integrales para la Mujer y de las seis que efectivamente tienen, sólo la Comuna 1 alcanza el objetivo de un CIM cada 50 mil habitantes mujeres.

Otra de las tendencias que identificamos, particularmente desde la asunción de la actual gestión de gobierno (diciembre 2015), es la tercerización de responsabilidades estatales a través de la figura de la gestión compartida. El CIM "Dignxs de ser” y la Casa de Medio Camino "Fundación Margarita Barrientos" (al igual de "La Casita Trans" recientemente inaugurada) son dispositivos creados desde el GCBA bajo la tercerización de la política pública a organizaciones no gubernamentales. La modalidad de intervención vía ONGs es una forma de incorporar en la agenda institucional las demandas del movimiento feminista y de mujeres, pero bajo una nueva forma de desvinculación estatal. La tercerización en ONGs de las políticas públicas, deberá ser material de un análisis riguroso. No sólo por la pérdida de globalidad que tiene una política pública descentralizada en agentes de la sociedad civil, sino también desde la perspectiva de los y las trabajadoras y de la continuidad de la política pública. 
Por otra parte, hemos identificado que las Casas de Medio Camino y los Refugios de Alta Seguridad son insuficientes. El Estado debe crear los dispositivos necesarios para que todas las mujeres que ingresan a los albergues con riesgos de vida mediano o alto, tengan resuelto al menos parcialmente el circuito de salida de la violencia. En este sentido, que las principales acciones del gobierno local frente a la violencia machista sean de asistencia, debería al menos abocarse a la construcción de casas de medio camino o refugios de alta seguridad, o al menos que los existentes cuenten con el presupuesto y el personal necesario para cumplir con los objetivos propuestos.

Asimismo, consideramos que la falencia más notable en cuanto prevenir y atender la violencia de género en la CABA, tiene que ver con la falta de integralidad a la hora de elaborar las políticas públicas. Teniendo en cuenta la complejidad de la problemática, las políticas contra la violencia de género concebidas para garantizar el acceso a derechos deben promover la autonomía económica, contemplar el hecho de que las mujeres son mayoritariamente quienes están a cargo de las tareas de cuidado e incorporar las condiciones de vida económicas, sociales y afectivas de las víctimas de violencia de género. Es decir, tienen que ser concebidas desde la perspectiva de género, lo que presupone elaborar, diseñar y poner en marcha acciones a largo plazo que relacionen con políticas locales de mayor alcance (Rodríguez Gustá, 2008). La falta de un abordaje integral puede observarse también en la falta de perspectiva que actualmente tienen los CIM: en cuatro de los siete existentes no hay trabajadora social. Esta ausencia expresa la carencia de una perspectiva socioambiental en el abordaje a la víctima, que se traduce en que muchas veces no sean contempladas las dificultades económicas y las tareas de cuidado que tienen muchas de las mujeres que se acercan a los CIM.

Además del deficiente abordaje de las víctimas por falta de profesionales especializados, la DGM desatiende otras áreas que dan cuenta de la falta de integralidad a la hora de plantear las políticas que de este organismo se desprenden. Con respecto a esto, no se constatan programas que se destinen a atender problemáticas relacionadas con el acceso a salud de las mujeres, o a la salud reproductiva y sexual, o con respecto a la situación laboral de las mujeres. Estas cuestiones deberían de ser contempladas por la DGM, teniendo en cuenta que consta de un Programa de Igualdad de Oportunidades, que podría estar en coordinación con otras áreas del gobierno local de manera tal de transversalizar la perspectiva de género en las políticas públicas. En este sentido, la falta de jerarquización por parte GCBA de la DGM se evidencia por ejemplo en el vaciamiento de personal que se lleva a cabo en el Observatorio de Equidad de Género, encargado de producir información sobre la realidad de las mujeres que viven en la Ciudad Autónoma de Buenos Aires y que hasta la fecha de redactarse este artículo no ha producido ningún informe. La dotación insatisfactoria de recursos se visibiliza en por falta de personal. La situación se debe al menos en parte a que el diagnóstico de "personal óptimo" que establece el GCBA muchas veces desconoce las particularidades del trabajo con población víctima de violencia de género. La asignación de personal a partir de cálculos aritméticos abstractos suele pasar por alto la nada infrecuente circunstancia de que para una sola víctima de violencia 
de género sea necesario tener disponible a tres o cuatro trabajadoras/es. Dicho problema no sólo se manifiesta en la falta de trabajadoras/es sociales en los CIM sino también en la falta de profesionales y facilitadoras en los albergues y casas de medio camino.

En relación a los y las trabajadoras de la DGM, hemos podido identificar el poco cuidado que existe para quienes cuidan. La planta trabajadora de la DGM se encuentra constantemente expuesta a situaciones de alta tensión y su situación laboral es precaria. Las formas de contratación y la insuficiente remuneración que perciben generan una alta situación de vulneración. A su vez, para cuidar a quienes cuidan, es necesaria la creación de espacios de "burn-out", es decir, espacios donde las trabajadoras puedan canalizar las emociones y las angustias que puede implicar el trabajo con situaciones de violencia. Las trabajadoras, además, plantean la necesidad de acceder a capacitaciones sobre perspectiva de género desde un enfoque integral, que les ayude a ir más allá de la asistencia y les dé herramientas para promover el empoderamiento de las mujeres víctimas de violencia de género.

Por otro lado, también queremos resaltar que la indagación respecto de los índices de violencia de género en la CABA y el diagnóstico de las políticas públicas existentes es una tarea que debería estar en la propia agenda del GCBA. Si bien la DGM cuenta con el Observatorio para la Equidad de Género ya mencionado, la falta de personal asignado a esta tarea deja como saldo una falta de información actualizada que permita monitorear adecuadamente las políticas públicas implementadas. Si no hay información, no se puede realizar un diagnóstico que pueda servir para mejorar las acciones que se estén llevando a cabo.

A esta altura, cabe señalar que la DGM no desarrolla programas destinados a la prevención de la violencia machista, ya que como podemos observar, no intenta promover campañas de concientización ni de educación en esta temática. Este es otro ejemplo de la falta de perspectiva integral para pensar las políticas públicas relacionadas con la violencia de género, ya que gran parte del problema requiere que se realicen acciones preventivas para evitar que se consumen las violencias más extremas.

\section{Consideraciones finales y desafíos para luchar contra la violencia de género}

En este trabajo nos propusimos identificar y diagnosticar el estado de situación de las políticas públicas destinadas a prevenir y erradicar la violencia de género. Para llevar a cabo el objetivo propuesto, en primer lugar, al no haber datos estadísticos que provengan de la GCBA y teniendo en cuenta los sesgos que implican los registros de la violencia a partir de denuncias, nos propusimos -por medio de estudios realizados por ONGs y organismos gubernamentales- cuantificar este fenómeno. En segundo lugar, observamos las políticas públicas destinadas a prevenir y atender a la violencia de género, con el fin de diagnosticar su funcionamiento y los marcos interpretativos en los cuales son formuladas. 
Al respecto, para empezar identificamos que es necesario otorgarle mayor centralidad y capacidad de acción a la Dirección General de la Mujer, no sólo al Programa de Asistencia a la Víctima, sino también al Programa Fortalecimiento de las Políticas de Igualdad de Oportunidades. Tal como fue mencionado a partir de la investigación de Pautassi y Rodríguez (2016) la restricción fiscal no puede ser una excusa válida para la inacción del Estado en materia de violencia de género, mucho menos en la CABA, que es una de las jurisdicciones con más recursos. En este sentido, consideramos que la decisión de jerarquizar este organismo debe ser política, a través de la traducción institucional de la creación de dispositivos que se encuentren centralizados por el Estado y no institucionalizados vía tercerización en ONGs, como ocurre en los últimos años.

Por otro lado, observamos que la vida de las víctimas de violencia de género se ve afectada en la totalidad de sus prácticas cotidianas, que se repliegan de sus otras relaciones sociales, pierden sus trabajos, están solas a cargo de sus hijos e hijas. Es por ello que vemos necesario respuestas integrales de las políticas públicas que trabajen con población víctima de violencia de género, ya que esto requiere una contundente política habitacional, políticas de generación de empleo e ingresos, disponibilidad de servicios de cuidado de los y las menores y adultos/as a cargo, acceso preferencial al sistema sanitario y espacios de contención y recreación que le permitan fortalecer lazos sociales y subjetivos desde una perspectiva de derechos y libres de violencias.

A lo largo de este artículo hemos intentado dar algunas explicaciones para poder comprender las principales falencias de las políticas públicas destinadas a la prevención y a la atención de las víctimas de violencia machista en la GCBA. A nuestro entender estas falencias provienen en primer lugar, como hemos dicho en el desarrollo de este estudio, del perfil fundamentalmente de asistencia que tienen estas políticas y de la falta de integralidad a la hora de planificar y elaborar iniciativas que vayan más allá de la atención y que puedan ser articuladas con otras áreas del gobierno local para no sólo atender, sino prevenir y erradicar, en un horizonte no muy lejano.

Entendemos que las políticas públicas deben ser elaboradas desde la perspectiva de género, que no considere a las mujeres como víctimas a ser asistidas, sino como sujetos de derechos. Estas políticas tienen que partir del reconocimiento de las relaciones desiguales entre los géneros para intervenir en pos de brindar a las mujeres, travestis y trans soluciones integrales no sólo a las violencias que afectan sus vidas sino el igual acceso a la ciudadanía. 
${ }^{1}$ Para ahondar en la construcción de cada una de las categorías, las baterías de preguntas están disponibles en el Informe Ejecutivo: http://contalaviolenciamachista.com/Informe-ejecutivo-final.pdf. Este estudio es el resultado de un trabajo de investigación, que por falta de recursos, no es una investigación probabilística. Sin embargo, para acceder a una muestra diversificada se llevaron adelante dos métodos de acceso a la población. Un primer método de acceso libre y voluntario a quien quisiera responder el cuestionario vía online y un segundo modo de acercamiento a la población a través de un muestreo intencional en zonas donde se asumía, el cuestionario virtual no había accedido. La muestra total país quedó conformada por 59.380 casos, y en la Ciudad Autónoma de Buenos Aires por 19.068 mujeres, travestis y trans respondientes.

${ }^{2} \mathrm{Al}$ respecto se han presentado diversos proyectos en la legislatura porteña, que no han sido tratados, que atacan la problemática de la violencia de género de manera integral tal como el Proyecto de ley para la asistencia integral de las víctimas de violencia de género, presentado por la Defensoría del Pueblo de la CABA. El proyecto hace hincapié en la definición de acciones para la asistencia integral de las víctimas y propone mejoras en los Centros Integrales de Atención que, de aprobarse, deberán contar con servicios de atención psicológica y tratamiento para las víctimas; asesoramiento y patrocinio jurídico gratuitos inmediatos, y asistencia social. Por otro lado, también se promueve un servicio de asistencia psicológica y tratamiento para los/as agresores/as, pero en días y horarios diferentes, para evitar el contacto con las víctimas, en los casos en que ello sea necesario.

${ }^{3}$ La DGM se creó el 8 de julio de 1989 como Subsecretaría de la Mujer y la Solidaridad Social. En el año 1992 pasó a depender de la Secretaría de Gobierno como Consejo, con rango de Subsecretaría. Esto le implicó elevarse a una estructura gubernamental con instancias decisionales intermedias y altas.

El proceso de autonomización de la Ciudad de Buenos Aires trajo consigo algunas modificaciones en el funcionamiento y estructura de la institución. A partir de 1994, el reciente Consejo volvió a constituirse como Dirección General bajo la órbita del Ministerio de Promoción Social de la Ciudad. Si bien y como detalláramos, la Dirección existía antes de la conformación de la Ciudad Autónoma de Buenos Aires, es en este momento que adquiere la fisonomía actual que implicó una transformación de la jerarquía en el organigrama, conformándose Coordinaciones y Programas sin una estructura administrativa que permita desarrollar líneas de trabajo articuladas e integrales.

${ }^{4}$ Según la autora, las políticas públicas de género, partiendo de una perspectiva que entiende que el género es un sistema de relaciones sociales (económicas, políticas, culturales) que determina las características y posiciones relativas de hombres, mujeres y trans, implica ampliar el marco de indagación más allá de los dispositivos focalizados, como es la Dirección General de la Mujer. En este sentido, Rofman hace la distinción entre las políticas explícitas e implícitas, siendo las primeras las que de forma deliberada pretenden incidir en las relaciones sociales entre los géneros y las segundas el conjunto de las intervenciones estatales, que de forma directa o indirecta cartografian las formas de socialización. El objeto de análisis de este trabajo es la política explícita llevada a cabo por la DGM.

${ }^{5}$ Según el censo 2010, la comuna 5 tiene 98.199 mujeres, la comuna 7: 118.110, la comuna 8: 97.692 y la comuna 11: 101.363. 


\section{Referencias bibliográficas}

Beck, I. y Romeo, M. (2016) "Informe ejecutivo. Argentina cuenta la violencia machista”. Disponible en: http://contalaviolenciamachista.com/Informe-ejecutivo-final.pdf

Chejter, S. (2009): "Violencia de género: modelos de abordaje.” En Chejter, S. Correa, V. Gimol, P. Abordajes frente a la violencia familiar desde una perspectiva de género e infancia: fortalecimiento de equipos de trabajo de género. Buenos Aires: Ministerio de Desarrollo Social de la Provincia de Buenos Aires.

ELA - GCBA (2016) “Las cifras de la violencia”. Disponible en: http://www.ela.org. ar/a2/index.cfm?fuseaction=MUESTRA\&codcontenido=2463\&plcontampl=6\&apli cacion $=\operatorname{app} 187 \& \mathrm{cnl}=14 \& \mathrm{opc}=49$

Femenías, M. L. y Rossi, P. (2009): "Poder y Violencia sobre el cuerpo de las mujeres". Sociologias 11 (21) 42-65.

Guzmán,V. (2001) La institucionalidad del género en el Estado: Nuevas perspectivas de análisis. Unidad Mujer y Desarrollo, CEPAL, Santiago de Chile. Disponible en: http://repositorio. cepal.org/bitstream/handle/11362/5878/S01030269_es.pdf?sequence=1\&isAllowed $=\mathrm{y}$

Hiller, R. y D. Jones (2016) “A seis años del matrimonio igualitario". Revista Bordes 1 (1) $179-185$.

Ministerio Público de la Defensa CABA (2016) Anuario de derechos económicos, sociales y culturales en la CABA 2015. Disponible en:https://www.mpdefensa.gob.ar/ biblioteca/pdf/Anuario_de_Derechos_Econ\%C3\%B3micos_Sociales_y_Culturales_ de_la_CABA_2015.pdf Ministerio Público Fiscal (2016). Femicidios y homicidios de mujeres en la Ciudad Autónomas de Buenos Aires en 2014. Informe diagnóstico y proyecto de relevamiento de casos de la UFEM. Disponible en: https://www.mpf.gob.ar/ufem/files/2016/09/ UFEM-Informe-Femicidios-y-Homicidios-de-Mujeres-en-CABA-2014.pdf

Morales, L. (2015). Derechos sociales, constitucionales y democracia. Madrid: Marcial Pons.

Mouffe, C.(1992) "Feminism, Citizenship, and Radical Democratic Politics “ en Judith Butler y Joan W. Scott, eds. Feminism theorize the Political. New York: Rouledge, pp. 369-385.

Oszlak, O. (2012) "La capilaridad social del rol del Estado". Voces en el Fénix, 3 (17).

Oszlak, O, y O`Donnell, G. (1982):"Estado y políticas estatales en América Latina: hacia una estrategia de investigación”, en Revista Venezolana de Desarrollo Administrativo N¹. 
Pautassi, L. y Rodríguez Enríquez, C. (2016) "Violencia contra las mujeres y políticas públicas. Implicancias fiscales y socioeconómicas” en Serie Asuntos de Género 137, ISSN 1564-4170, Repositorio CEPAL. Disponible en: http://repositorio.cepal.org/ bitstream/handle/11362/40483/6/S1600701_es.pdf

Rodríguez Gustá, A. L. (2008): "Las políticas sensibles al género: variedades conceptuales y desafios de intervención”. Revista Temas y debates 12 (16), 109-130.

Rofman, A. (2000) “El Estado y las mujeres: articulación entre demandas y políticas de género" en Hintze, Susana (comp.) Estado y sociedad. Las políticas sociales en los umbrales del siglo XXI. Colección CEA. Buenos Aires: Eudeba.

Sagot, M. (2008): “Los límites de las reformas:Violencia contra las mujeres y políticas públicas en América Latina”. Revista Ciencias Sociales 120.

Segato, Rita (2003) Las estructuras elementales de la violencia. Ensayos sobre género entre la antropología, el psicoanálisis y los derechos humanos. Buenos Aires: Universidad Nacional de Quilmes. Prometeo.

\section{Como citar este artículo}

Penchansky, Celina, Lucía Fuster Pravato, Victoria Freire y Lucía Loewy (2018) “¿Prevenir, atender y erradicar?: políticas públicas contra la violencia de género en la Ciudad de Buenos Aires, 2015 - 2017”. Revista Perspectivas de Políticas Públicas vol 7 №14:297-321 